\title{
The Impact of Variable Fluid Properties on Biomagnetic Maxwell Fluids Past a Stretching Sheet with Slip Velocity and Heat Generation/Absorption
}

\author{
M. G. Murtaza', M.Z.I. Bangalee ${ }^{2}$, Mohammad Sahadet Hossain ${ }^{3}$ and M. Ferdows ${ }^{2 *}$ \\ ${ }^{1}$ Department of Mathematics, Comilla University, Cumilla-3506, Bangladesh. \\ ${ }^{2}$ Department of Applied Mathematics, University of Dhaka, Dhaka-1000, Bangladesh. \\ ${ }^{3}$ Department of Mathematics and Physics, North South University, Dhaka-1229, Bangladesh.
}

(Received : 11 July 2021; Accepted : 14 October 2021)

\begin{abstract}
The impact of variable fluid properties (viscosity and thermal conductivity) and magnetic dipole on biomagnetic Maxwell fluid past a stretching sheet with slip velocity and heat generation/absorptionhave been studied. Similarity transformation technique is adopted to obtain the self-similar coupled nonlinear ordinary differential equations. Using similarity variable, the basic governing equations with boundary conditions are transformed and solved in bvp4c technique with MATLAB software. The contribution of different pertinent parameters such as viscosity, thermal conductivity and ferromagnetic parameter on the flow profiles with physical quantities are analyzed and examined through graphically. Results shown that with increasing ferromagnetic parameter, slip parameter, Maxwell parameter, velocity decreases but temperature increases. For accuracy of the proposed model to compare our numerical results in numerically and graphically with the previousliterature under some limiting cases and a good agreement is found.
\end{abstract}

Keywords: Biomagnetic fluid, ferrofluid, Maxwell fluid, magnetization, stretching sheet.

\section{Introduction}

Biomagnetic fluid dynamics is an interdisciplinary field comprising engineering, medicine and biology. Some of the living body related infections and disorders are finding and curing from the developing bio fluid dynamics. Biomagnetic fluid dynamics (BFD) namely namely biofluid dynamics is the base of knowledge of magnetrohydrodynamics (MHD) and ferrohydrodynamics (FHD) which deals with the magnetization force and Lorentz force. In biomagnetic fluid dynamics Haik et al. ${ }^{1}$ first introduced the mathematical model by considering the applied magnetic field.Further Tzirtzilakis ${ }^{2}$ extended Haik et al. ${ }^{1}$ model where they introduce theFHD and MHD of their model. Tzirtzilakis and Kafoussias ${ }^{3}$, Murtaza et al. $^{4-5}$ presented the mathematical model where they described the biomagnetic fluid past a linearly stretching sheet.

Maxwell fluid is a one kind of viscoelastic fluid which exists the both properties of viscosity and elasticity of a fluid. One the other hand, the fluid (blood) shows shearrate dependent viscosity and thus cannot be proposed the Newtonian fluids model and different models of nonNewtonian fluids have been suggested by many investigators. So many researcher investigated of Maxwell fluid due to the applications of biological transportation, drug delivery, high-temperature plasmas, power generation and cancer therapy and so on. Some of them, Rashid et al. ${ }^{6}$ analyzed the behaviour for rotating Maxwell nanomaterial flow and showed the progress the thermalbase material. Khan et al. ${ }^{7}$ performed the Maxwell fluid flow with containing motile microorganisms and nanoparticles. Sadiq and Hayat ${ }^{8}$ investigated the MHD Maxwell fluid with moving surface. Afify and Elgazery ${ }^{9}$ showed the effect of chemical reaction on MHD Maxwell nanofluid with a stretching sheet. Abbasi and Shehzad ${ }^{10}$ analyzed the variable fluid properties of Maxwell fluid. Sajid et al. ${ }^{11}$ reported the impact of slip effect on Maxwell fluid. Gayatri et al. ${ }^{12}$ analyzed the Melting heat transfer with heat source effect on Maxwell fluid. Shehzad et al. ${ }^{13}$ presented the hydromagnetic flow of Maxwell fluid. Analyzed the impact of thermophoretic properties and magnetic dipole are presented by Kumar et al. ${ }^{14}$ Murtaza et al. ${ }^{21}$ investigated the 3D biomagnetic Maxwell fluid past a linear stretched sheet.

From the analysed of previous published work, we concluded that a very little concentration about nonNewtonian fluids with slip flow effect. Some of the reputed work about Maxwell fluid are cited here. Hayat et al. ${ }^{15}$ examined the slip effect on Maxwell fluid. Sajid et al. ${ }^{16}$ examined the influence slip flow rate on Maxwell fluid and Lin and $\mathrm{Guo}^{17}$ have performed slip velocity flows of Maxwell fluids.

From the literature survey we seem that there is no available existing literature in which the effects of variable fluid property in biomagneticMaxwell fluid with the slip boundary condition and heat generation/ absorption. In view of this fact the problem of slip flow of a Maxwell fluid is considered in this paper.

\section{Problem Formulation}

We analysis the Maxwell fluid which is incompressible, laminar and electrically non conducting. For the flow geometry we take the coordinate system (see fig 1) where the flow direction shows the $\mathrm{x}$ axis and $\mathrm{y}$ axis perpendicular to it. Consider $u=a x$ is the surface velocity. The strength magnetic field $B_{0}$ which is act in perpendicular to the flow direction. The flow is influenced by the magnetic field which is created from magnetic dipole. 


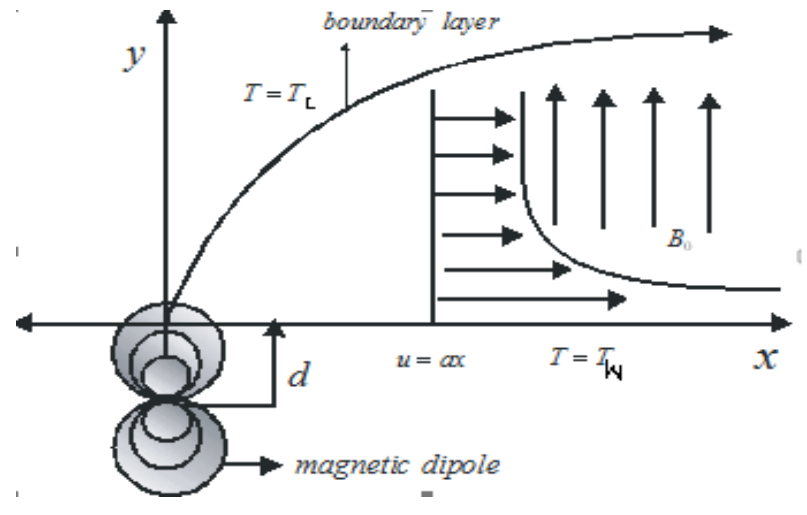

Fig. 1. Physical model and coordinate system of the problem

Based on the above assumptions, the basic governing equations can be expressed as follows by Loganathan et al. $^{22}$ :

$$
\frac{\partial u}{\partial x}+\frac{\partial v}{\partial y}=0
$$

$u \frac{\partial u}{\partial x}+v \frac{\partial u}{\partial y}=\frac{1}{\rho_{\infty}} \frac{\partial}{\partial y}\left(\mu \frac{\partial u}{\partial y}\right)+$

$\frac{1}{\rho_{\infty}} \mu_{0} M \frac{\partial H}{\partial x}-\tau\left[u^{2} \frac{\partial^{2} u}{\partial x^{2}}+v^{2} \frac{\partial^{2} u}{\partial y^{2}}\right.$

$\left.+2 u v \frac{\partial^{2} u}{\partial x \partial y}\right]-\frac{\sigma B_{0}^{2}}{\rho_{\infty}} u$

$\rho_{\infty} c_{p}\left[u \frac{\partial T}{\partial x}+v \frac{\partial T}{\partial y}\right]+\mu_{0} T \frac{\partial M}{\partial T}\left(u \frac{\partial H}{\partial x}+\right.$

$\left.v \frac{\partial H}{\partial y}\right)=\frac{\partial}{\partial y}\left(k \frac{\partial T}{\partial y}\right)+Q_{0}\left(T_{c}-T\right)$

and the basic boundary conditions are:

$u=U+\gamma \frac{\partial u}{\partial y}, v=-v_{w}, T=T_{w} \quad$ at $\quad y=0$

$u \rightarrow 0, T \rightarrow T_{c} \quad$ at $\quad y \rightarrow \infty$

Here $x, y$ are the Cartesian coordinates, $u, v$ denotes the velocities in $x$ and $y$ axes, $v$ depicts the kinematics viscosity, $\rho_{\infty}$ indicates the density, $\mu_{0}$ is the magnetic permeability, $k$ denotes the thermal conductivity. $Q_{0}$ indicates the coefficient heat generation/absorption, $\sigma$ denotes the electrical conductivity, $\tau$ indicates the relaxation time and the following relation used by Murtaza et al. ${ }^{4}$

$M=K H(T c-T)$
Where $\mathrm{H}$ is a magnetic field intensity, can be expressed by analogous manner, as

$H(x, y)=\frac{\gamma}{2 \pi}\left[\frac{1}{(y+d)^{2}}-\frac{x^{2}}{(y+d)^{4}}\right]$

\section{Mathematical Analysis}

For simplify the dimensionless we introducing the following similarity transformations

$$
\begin{aligned}
& \eta=\sqrt{\frac{a}{v} y, u=a x f^{\prime}(\eta)} \\
& v=-\sqrt{a v} f(\eta), \theta=\frac{T c-T}{T c-T w}
\end{aligned}
$$

consider that viscosity of the fluid is exponential form where thermal conductivity is linear form Manjunathaa et al. $^{20}$

$\mu=\mu_{\infty} e^{-a \theta} \quad$ and $\quad k=k_{\infty}(1+\varepsilon \theta)$

Where $\alpha$ denotes variable fluid viscosity parameter, $\varepsilon$ denotesvariable thermal conductivity parameter, where $\mu_{\infty}$ and $k_{\infty}$ indicates the viscosity and thermal conductivity of the fluid afar from the sheet respectively. It general $\alpha>0$ for liquid and $\alpha<0$ for gases ${ }^{20}$.

Rewriting the equations (2)-(4) with the help of (5)-(8):

$$
\begin{aligned}
& f^{\prime \prime \prime}-\alpha f^{\prime \prime} \theta^{\prime}+e^{\alpha \theta}\left(f f^{\prime \prime}-f^{\prime 2}-M f^{\prime}-\right. \\
& \frac{\beta \theta}{(\eta+\delta)^{4}}+A\left(2 f f^{\prime} f^{\prime \prime}-f^{2} f^{\prime \prime}\right)=0 \\
& (1+m \theta) \theta^{\prime \prime}+m \theta^{\prime 2}+\operatorname{Pr}\left(f \theta^{\prime}+\lambda_{1} \theta\right)- \\
& \frac{\delta^{2} \lambda \beta(\varepsilon-\theta) f}{(\eta+\delta)^{3}}=0
\end{aligned}
$$

With boundary conditions (5) are

$$
\begin{aligned}
& f(0)=s, f^{\prime}(0)=1+\gamma f^{\prime \prime}(0), \theta(0)=1 \\
& f^{\prime}(\infty) \rightarrow 0, \theta(\infty) \rightarrow 0
\end{aligned}
$$

$P_{r}=\frac{\mu_{\infty} c_{p}}{k_{\infty}} \quad$ denote the Prandtl number, $\lambda=\frac{a \mu^{2}}{\rho_{\infty} k_{\infty}\left(T_{c}-T_{w}\right)}$ indicate the viscous dissipation parameter and dimensionless Curie temperature, $\quad \varepsilon=\frac{T_{c}}{T_{c}-T_{w}}$, ferromagnetic

interaction parameter,

$$
\beta=\frac{\gamma}{2 \pi} \frac{\mu_{0} K\left(T_{c}-T_{w}\right) \rho}{\mu^{2}}
$$

dimensionless distance, $\delta=d \sqrt{\frac{a}{v}}$, Magnetohydrodynamic 
parameter, $M=\frac{\sigma}{\rho_{\infty} a} B_{0}$, Deborah number $A=a \tau, S$ is a suction/injection parameter and $\gamma$ is a velocity slip arameter. $\lambda_{1}=\frac{Q_{0}}{\rho c_{p}}$ is the heat generation/absorption parameter.

\section{Numerical Method}

By using bvp $4 c$ solver technique, the equations (9) to (11) are solve. For purpose of this technique first we reduce a system of first order ODE by setting $f=y_{1}, f^{\prime}=y_{2}, f^{\prime \prime}=y_{3}, \theta=y_{4}, \theta^{\prime}=y_{5}$

Then the equations (9) to (11) can be rewritten as given below

$$
\left.\begin{array}{c}
f^{\prime}=y_{2} \\
f^{\prime \prime}=y_{2}^{\prime}=y_{3} \\
f^{\prime \prime \prime}=y_{3}^{\prime}=\alpha y_{3} y_{5}-\exp \left(\alpha y_{4}\right)\left(y_{1} y_{3}-y_{2}^{2}-\right. \\
M y_{2}-\frac{\beta y_{4}}{(\eta+\delta)^{4}}+A\left(2 y_{1} y_{2} y_{3}-y_{1}^{2} y_{3}\right) \\
\theta^{\prime}=y_{5} \\
\theta^{\prime \prime}=\mathrm{y}_{5}^{\prime}=\left(\frac{1}{1+m y_{4}}\right)\left(-\operatorname{Pr}\left(y_{1} y_{5}+\lambda_{1} y_{4}\right)-\right. \\
m y_{5}^{2}+\frac{2 \lambda \delta^{2} \beta\left(\varepsilon-y_{4}\right) y_{1}}{(\eta+\delta)^{3}}
\end{array}\right\}
$$

and boundary conditions:

$$
\begin{aligned}
& y_{1}(0)=S, y_{2}(0)=1+\gamma y_{3}, y_{4}(0)=1, \\
& y_{2}(\infty)=0, y_{4}(\infty)=0 .
\end{aligned}
$$

All these simplifications are done for using the MATLAB package.

The numerical procedure of bvp4c followed is:

$>$ Nonlinear PDEs are reduced to $1^{\text {st }}$ order ODEs .

$>$ The solution is returned by bvp $4 \mathrm{c}$ as a structure called sol

$>$ Mesh selection is generated and returned in the field sol.x

Solution can be fetch from array sol.y corresponding to sol.x

let $y(0)$ be the left boundary, $y(\infty)$ be the right boundary

\section{Results and Discussion}

The numerical computations are presented in different forms through the graph for several values relevant parameter. For the numerical computation we required to assign some numerical values for entering into the problem.

For this, we can assume ${ }^{4}$ that $\rho=1050 \mathrm{kgm}^{-3}$, $\mu=3.2 \times 10^{-3} \mathrm{kgm}^{-1} \mathrm{~s}^{-1}, \quad \sigma=0.8 \mathrm{sm}^{-1}$. The temperature field is adopted under the influence of the applied magnetic field. The temperature of the fluid $T_{c}=41^{0} C_{\text {while the plate temperature }} T_{c}=37^{\circ} C_{\text {[4].So }}$ the dimensionless temperature is $\varepsilon=78.5$ and the viscous dissipation number $6.4 \times 10^{-14}$. Also we assumed that $c_{p}=3.9 \times 10^{3} \mathrm{Jkg}^{-1} \mathrm{~K}^{-1}$ and $k=0.5 \mathrm{Jm}^{-1} \mathrm{~s}^{-1} \mathrm{~K}^{-1}$ and hencethe prandtl number is determine and which is equal to $\operatorname{Pr}=25$

As far as the ferromagnetic parameters arise from the magnetic dipole and in the presentstudy we set the value of $\beta$ to be 0 to10. Moreover, Magnetohydrodynamics parameter $M$ also adopted which is 0 to 10 .

Table I. Comparison of $-f$ " $(0)$ withseveral values of slip parameter when $S=M=\alpha=\beta=A=0$

\begin{tabular}{|l|l|l|}
\hline$\gamma$ & Anderson [19] & Present result \\
\hline 0.0 & 1.000 & 0.999998 \\
1.0 & 0.4302 & 0.430158 \\
2.0 & 0.2840 & 0.283981 \\
5.0 & 0.1448 & 0.144842 \\
\hline
\end{tabular}

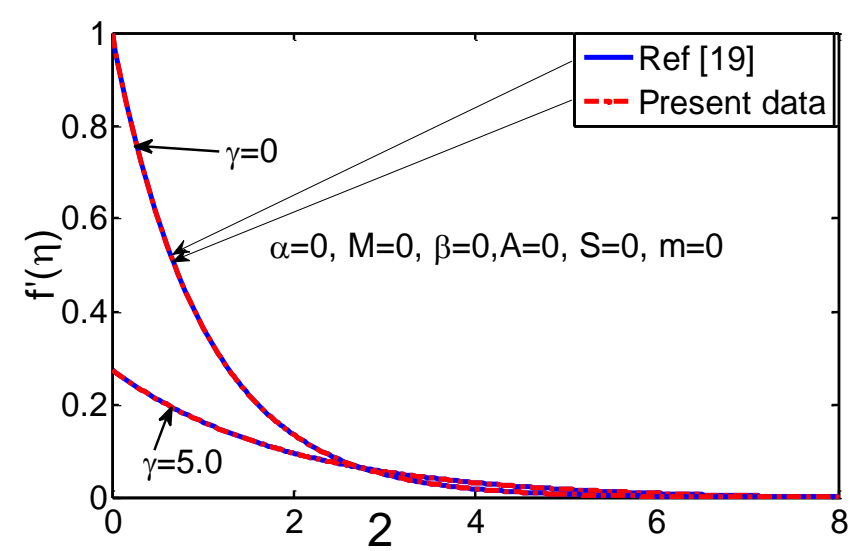

Fig. 2. Comparison of velocity profile with several values of slip parameter $\gamma$.

For validity of the numerical method in this study, First, we compare our results the coefficient of skin friction and velocity profile with Anderson [19] (see Table I) and figure 2 and observed that a good agreement is found which gives confidence that our results are accurate. 


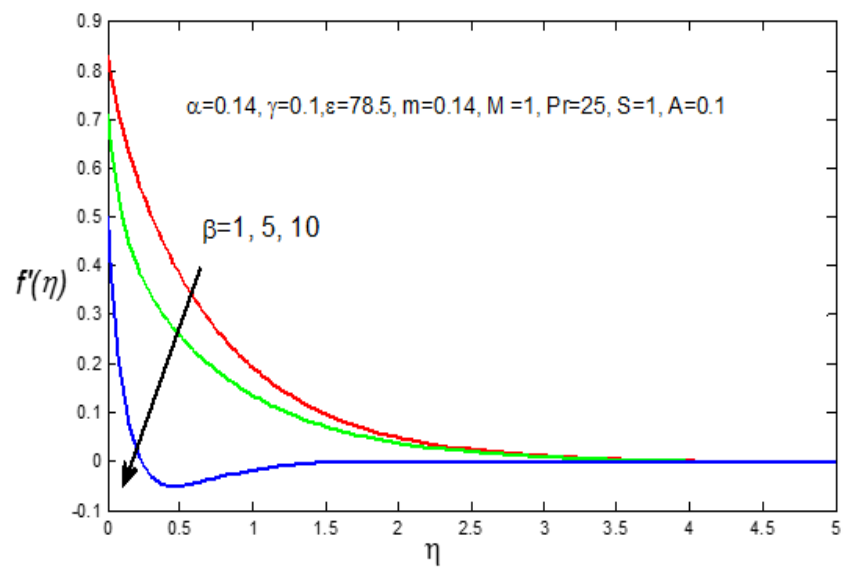

Fig. 3. $f^{\prime}(\eta)$ for numerous value of ferromagnetic parameter $\beta$

Figs. 3 and 4, clearly show the impact of ferromagnetic number on the dimensionless velocity distribution and temperature distribution. From Fig. 3, it is observed thatthe fluid velocityreduces with the increase the ferromagnetic parameter $\beta$. This is happening due to ferromagnetic number increases which tends to the growing upof Lorentz force, as a result resistance to the velocity transport phenomena. It is also noticed that with increasing ferromagnetic parameter to enhances the temperature profiles. This can be recognized to the fact that more Lorentz force produceswhich compete beside the fluid motion, hence heat is produced and summary of the result, temperature boundary layer thickness goes thicker for heavier magnetic field.

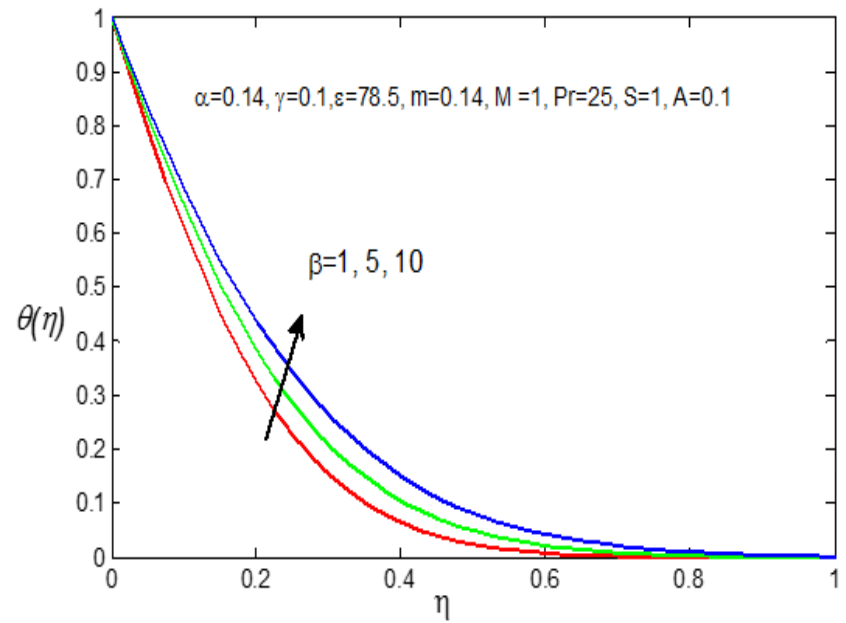

Fig. 4. $\theta(\eta)$ for numerous value of ferromagnetic parameter $\beta$

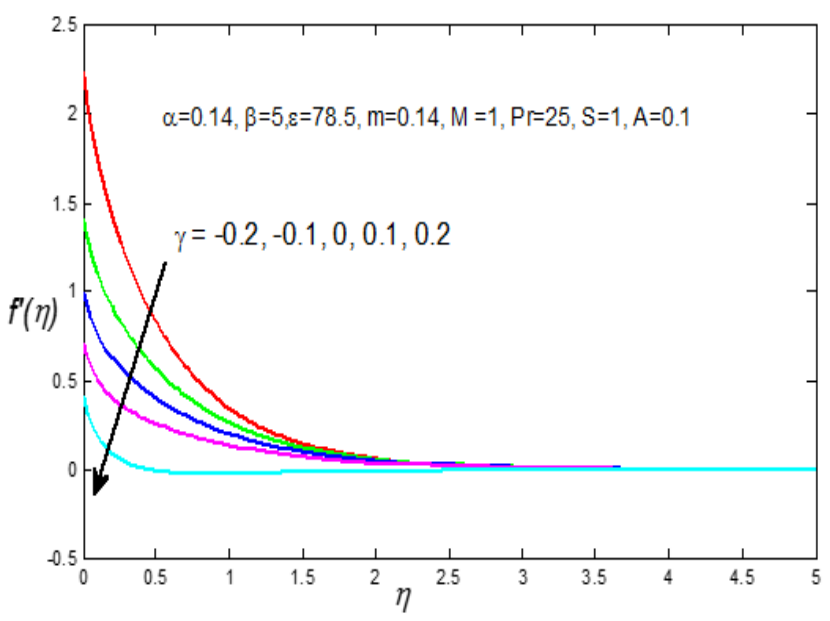

Fig. 5. $f^{\prime}(\eta)$ for numerous value of slip parameter ${ }^{\gamma}$.

Figs. 5 and 6 show that the effect of slip parameter on $f^{\prime}(\eta)$ and $\theta(\eta)$. From these figure, it is observed that an increase in the

slip parameter tends to decrease the velocity of the fluid.

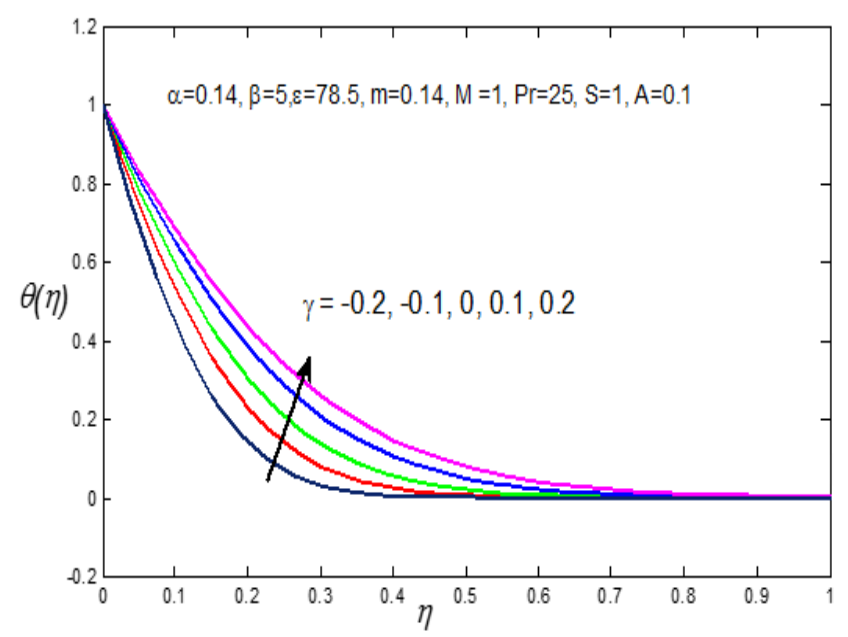

Fig. 6. $\theta(\eta)$ for numerous value of slip parameter ${ }^{\gamma}$

Slip velocity arises at the interface between the fluid and solid and it decrease monotonically tends to zero far away from the surface. When no slip condition occurs then the fluid velocity neighbouring to the surface is equal to the stretching velocity, which is fulfil the boundary condition see the figure 5. Figure 6 point out that temperature increase with growing velocity slip parameter.

Figs 7 and 8 show the effect of temperature dependent viscosity parameter on $f^{\prime}(\eta)$ and $\theta(\eta)$. It is noticed that for growing viscosity parameter, the velocity 


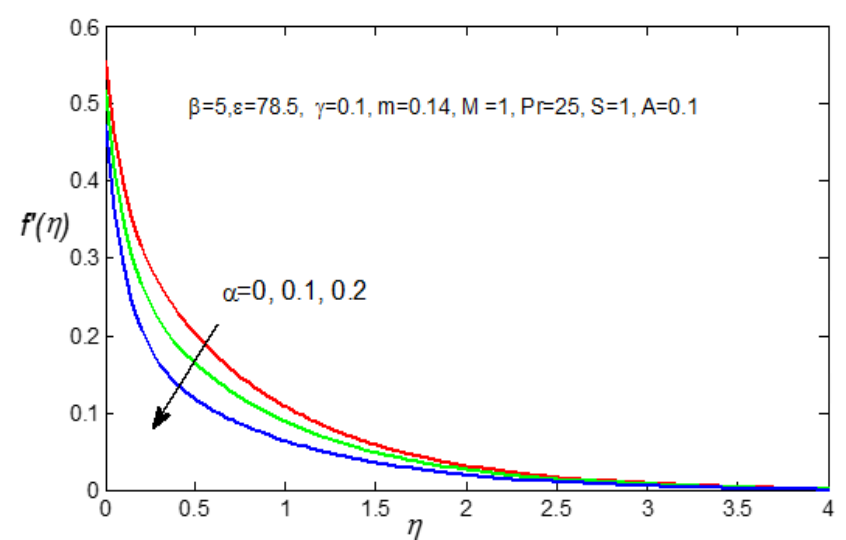

Fig. 7. $f^{\prime}(\eta)$ for numerous value of viscosity parameter $\alpha$

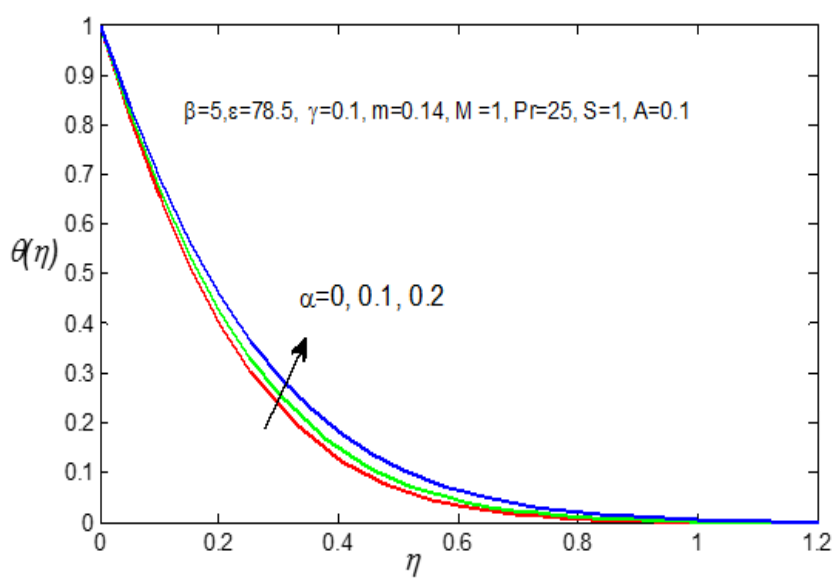

Fig. 8. $\theta(\eta)$ for numerous value of viscosity parameter $\alpha$.

distribution reduces but in this case the temperature distribution increases. This happen because viscosity growing up from the more internal frictional force or resistance. That's why for developing viscosity corresponds to the rising the resistance to flow which diminished the velocity and induce the temperature of the fluid which leads to induce the skin friction and reduce in the rate of heat transfer from fluid to the surface (see figure 13 and 14).

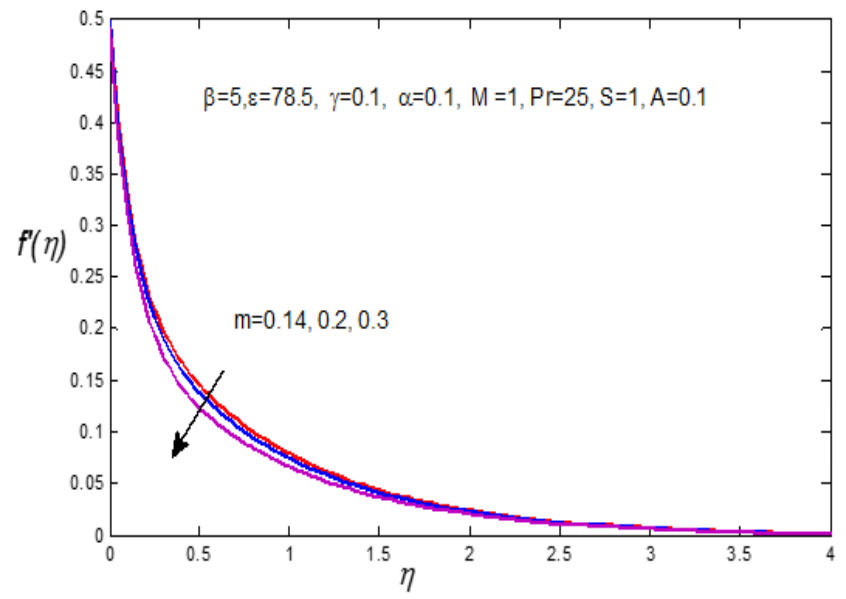

Fig. 9. $f^{\prime}(\eta)$ for numerous value of thermal conductivity parameter $m$.

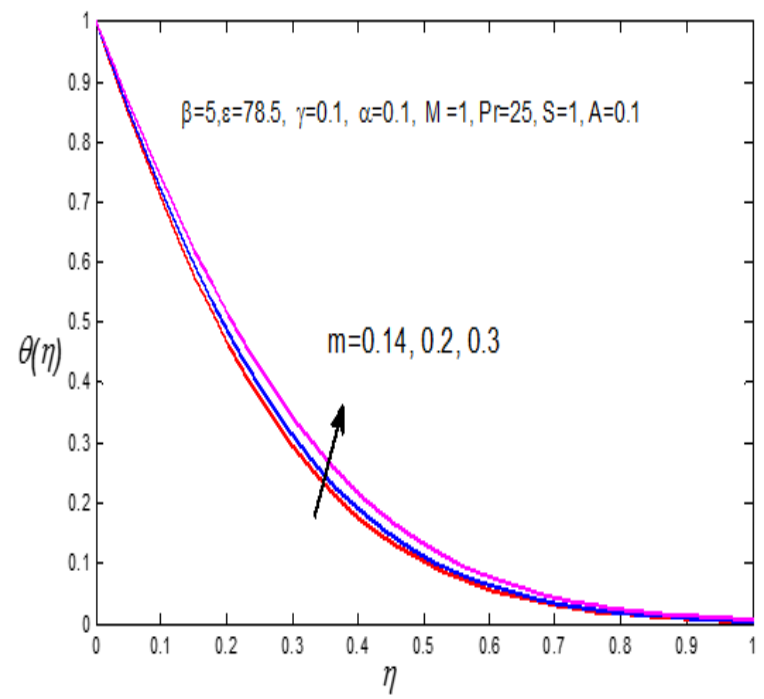

Fig. 10. $\theta(\eta)$ for numerous value of thermal conductivity parameter $m$.

The influence of thermal conductivity parameter $(m)$ on $f^{\prime}(\eta)$ and $\theta(\eta)$ areanalyzed in Figs. 9 and 10. From these figure, it can be seen that the thermal conductivity increases the velocity slightly decreases whereas the temperature distribution increases. This is fact due to the thermal conductivity parameter, the boundary layer to produce force which grounds the temperature increases.

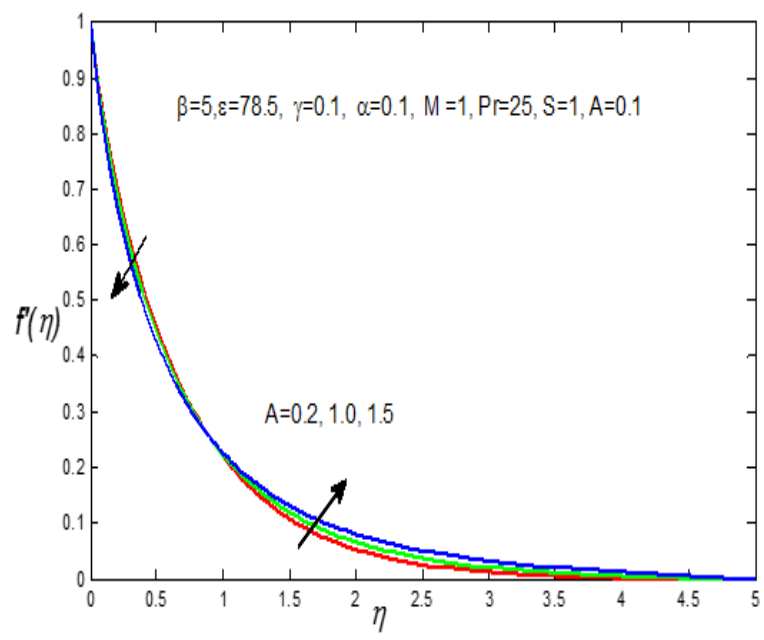

Fig. 11. $f^{\prime}(\eta)$ for numerous value of Maxwell parameter $A$.

The influence of Maxwell parameter on $f^{\prime}(\eta)$ and $\theta(\eta)$ are clearly exhibits in figures 11 and 12 . It is shown that increase of Maxwell parameter velocity reduces near the sheet but afar from the sheet (i.e. approximately at point $\eta=0.2)$ the profile overlap and then increases. This behaviour occurs because within the increase of relaxation time increases as a result both velocity profiles and boundary layer thickness decrease near the sheet and increases far away the sheet. 


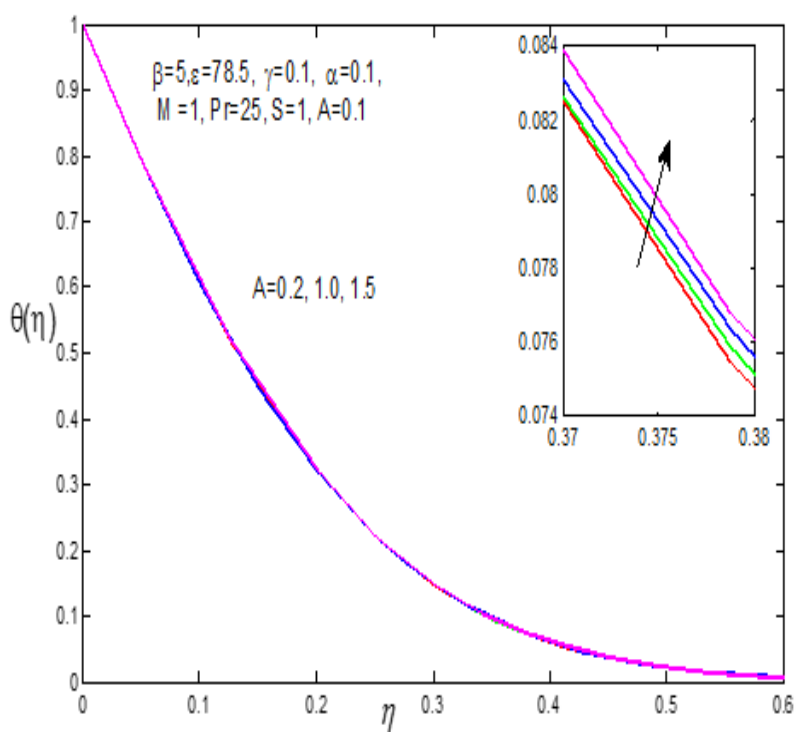

Fig. 12. $\theta(\eta)$ for numerous value of Maxwell parameter $A$.

Figure 12 show the effect of Maxwell parameter on the temperature profiles above the sheet. With increasing the Maxwell parameter is seen to reduces the fluid temperatureover the sheet.

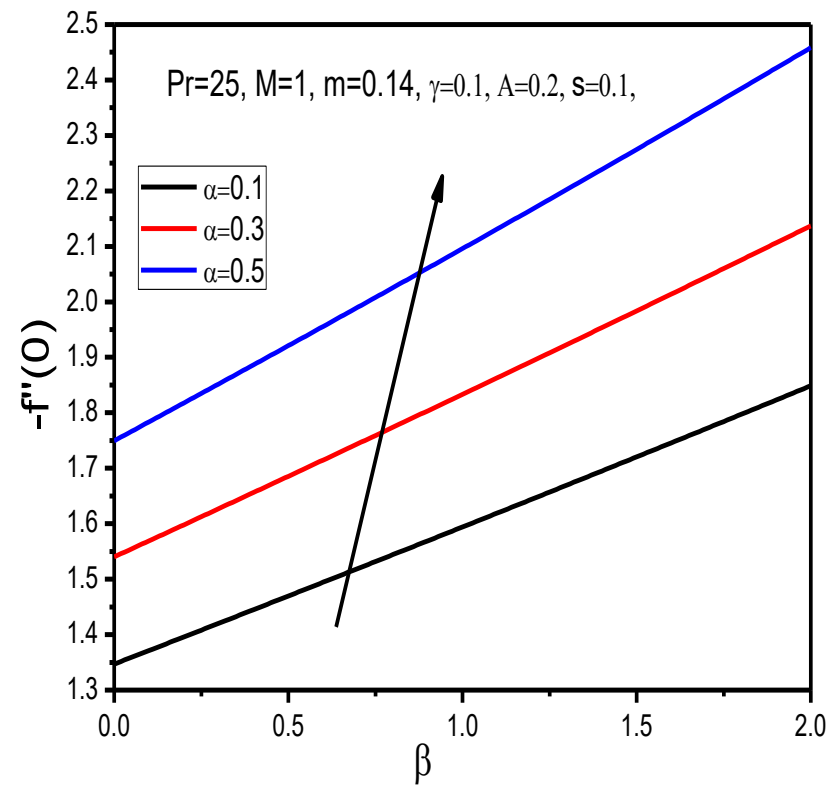

Fig. 13. $f^{\prime \prime}(0)$ with $\beta$ for numerous values of $\alpha$.

From figure 13 to 16 shows the effect of various values of ferromagnetic, viscosity and slip parameter on $f^{\prime \prime}(0)$ and $\theta^{\prime}(0)$.From these figure, we concluded that with increasing ferromagnetic parameter $f^{\prime \prime}(0)$ increases while $\theta^{\prime}(0)$ are decreases. Also we observed that for increases viscosity parameter, $f^{\prime \prime}(0)$ is also increases while $\theta^{\prime}(0)$ are decreases but the opposite behaviour have shown with slip parameter.

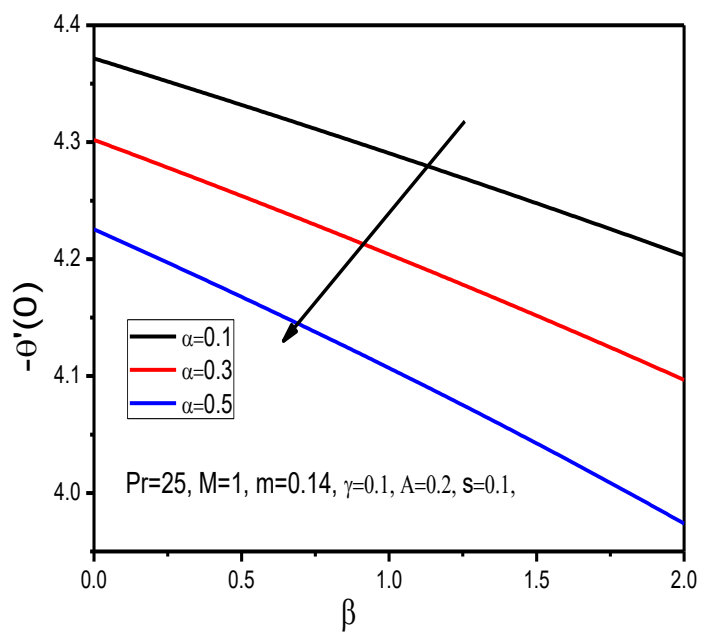

Fig. 14. $\theta^{\prime}(0)$ with $\beta$ for numerous values of $\alpha$

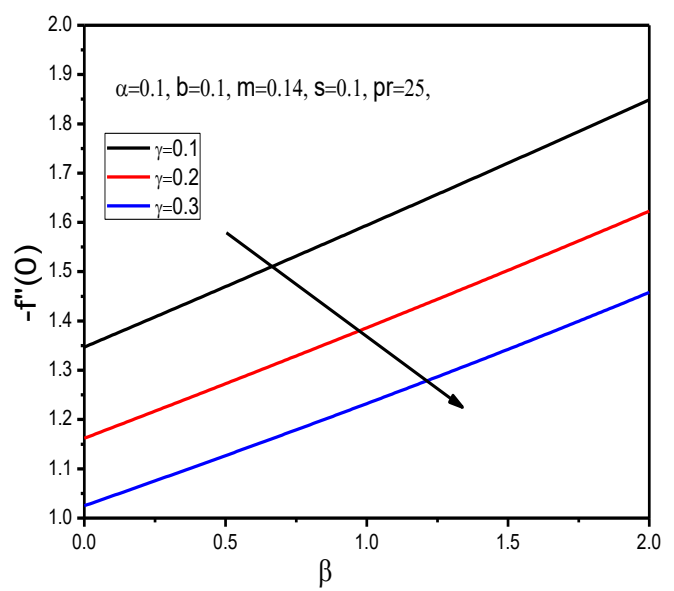

Fig. 15. $f^{\prime \prime}(0)$ with $\beta$ for numerous values of $\gamma$.

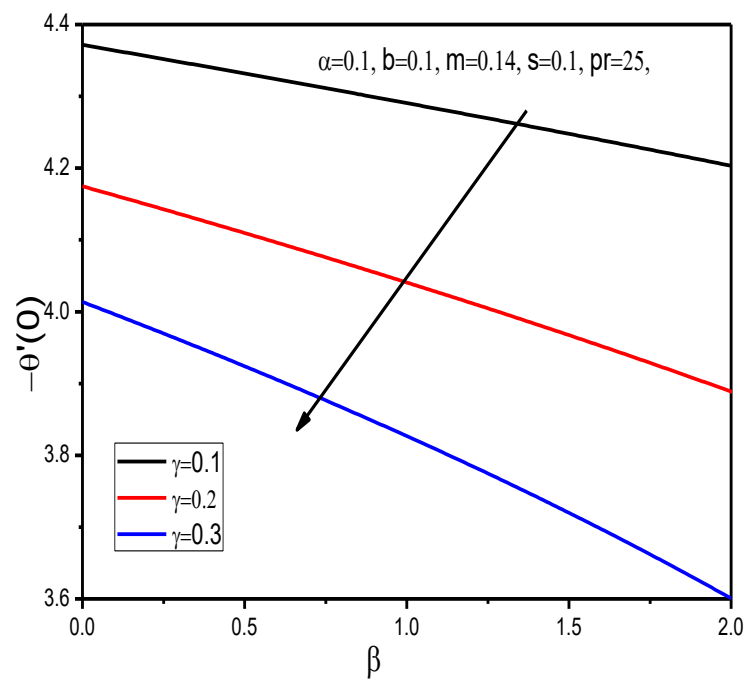

Fig. 16. $\theta^{\prime}(0)$ with $\beta$ for numerous values of $\gamma$ 


\section{Conclusion}

The main conclusions of this analysis can be précised as follows.

1. With increasing slip parameter, $f^{\prime}(\eta)$ decreasesbut $\theta(\eta)$ increases.

2. Maxwell parameter leads to decrease the velocity but increase the temperature profile.

3. With Ferromagnetic parameter supports to induce the temperature distribution and reduce the velocity profile.

4. The skin friction increases and heat transfer coefficient decreases with increasing ferromagnetic parameter.

5. Effect of viscosity parameter is to decrease the velocity profile, whereas the skin friction coefficient is increases.

6. For increasing values of thermal conductivity parameter, velocity reduces but the temperature induces.

\section{References}

1. Haik Y, Pai V and Chen C J.,1999. Biomagnetic fluid dynamics, in fluid dynamics at interfaces. edited by $\mathrm{W}$. Shyy and R. Narayanan (Cambridge University Press, Cambridge), 439-452.

2. Tzirtzilakis EE. 2005.A Mathematical model for blood flow in magnetic field.Physics of Fluids, 17(7), 077103-1-14.

3. Tzirtzilakis EE and Kafoussias NG. 2003. Bio-magnetic fluid flow over a stretching sheet with nonlinear temperature dependent magnetization. Z. angew. Math. Phys. 54, 551565 .

4. Murtaza MG, Tzirtzilakis EE, Ferdows M. 2017. Effect of electrical conductivity and magnetization on the biomagnetic fluid flow over a stretching sheet. Z. Angew. Math, Phys (ZAMP).68, 93 DOI 10.1007/s00033-017-0839$z$

5. Murtaza MG, Tzirtzilakis EE and Ferdows M. 2018. Numerical solution of three dimensional unsteady biomagnetic flow and heat transfer through stretching/shrinking sheet using temperature dependent magnetization, Archives of mechanics, 70 (2), 161-185.

6. Sadia Rashid, Ijaz MK, Hayat T, Ayub M and Alsaedi, A. 2019. Numerical treatment for rotating Maxwell nanomaterial flow with Arrhenius energy, Applied Nanoscience. 1-8. https://doi.org/10.1007/s13204-01900998-3

7. Khan MI, Waqas M, Hayat T, Imran Khan M, Alsaedi A.2017. Behavior of stratification phenomenon in flow of Maxwell nanomaterial with motile gyrotactic microorganisms in the presence of magnetic field. Int $J$ Mech Sci.131, 426-434.

8. Sadiq MA, Hayat T. 2016. Darcy-Forchheimer flow of magneto Maxwell liquid bounded by convectively heated sheet. Results Phys.6, 884-890.

9. Afify AA, Elgazery NS.2016. Effect of a chemical reaction on magneto hydrodynamic boundary layer flow of a
Maxwell fluid over a stretching sheet with nanoparticles. Particuology.29, 154-161.

10. Abbasi FM, Shehzad SA. 2016. Heat transfer analysis for three-dimensional flow of Maxwell fluid with temperature dependent thermal conductivity: application of CattaneoChristov heat flux model. J Mol Liq.220, 848-854

11. Muhammad Sajid, Zaheer Abbas, Nasir Ali,Tariq Javed, Iftikhar Ahmad, 2014. Slip Flow of a Maxwell Fluid Past a Stretching Sheet, Walailak J.Sci \&Tech,11(12), 1093-1103.

12. Mocherla Gayatri, Konda Jayaramireddy, Macherla Jayachandra Babu,2019. Nonlinear Convective Flow of Maxwell Fluid over a Slendering Stretching Sheet with Heat Source/Sink, J. Appl. Comput. Mech., 6, doi: 10.22055/jacm.2019.31394.1868

13. Shehzad SA, Alsaedi A, Hayat T. 2013. Hydromagnetic Steady Flow of Maxwell Fluid over a Bidirectional Stretching Surface with Prescribed Surface Temperature and Prescribed Surface Heat Flux. PLoS ONE 8(7), e68139. doi:10.1371/journal.pone.0068139

14. R. Naveen Kumar, A.M. Jyothi, Hesham Alhumade, R.J. Punith Gowda, Mohammad Mahtab Alam, Irfan Ahmad, M.R. Gorji, B.C. Prasannakumara, 2021. Impact of magnetic dipole on thermophoretic particle deposition in the flow of Maxwell fluid over a stretching sheet, Journal of Molecular Liquids,334, 116494.

15. Hayat T, Hina S, Hendi AA. 2010. Slip effects on the magnetohydrodynamic peristaltic flow of a Maxwell fluid, Z. Naturforsh. 65,1123-1136.

16. Sajid M, Abbas Z, Ali N, Javed T, Ahmad I.2014. Slip flow of a Maxwell fluid past a stretching sheet. Walailak Journal of Science and Technology. 11(12), 1093-1103.

17. Lin Y and Guo B.2017. Effect of second-order slip on the flow a fractional Maxwell MHD fluid. Journal of the Association of Arab Universities for Basic and Applied Sciences. 24, 232-241.

18. Mahmoud MAA 2011. The Effects of Variable Fluid Properties on MHD Maxwell Fluids Over a Stretching Surface in the Presence of Heat Generation/Absorption. Chem. Eng. Comm., 198,131-146.

19. Andersson HI , 2002.: Slip flow past a stretching surface. Acta Mech. 158, 121-125.

20. Manjunatha, S., Gireesha, B.J., 2016. Effects of variable viscosity and thermalconductivity on MHD flow and heat transferof a dusty fluid, Ain Shams Engineering Joutrnal,7(1), 505-515.

21. Murtaza, M. G., Ferdows, M., Misra, J. C., Tzirtzilakis, E.E., 2019. Three-dimensional biomagnetic Maxwell fluid flow over a stretching surface in presence of heat source/sink, International Journal of Biomathematics,12(3),1950036-20.

22. Loganathan, K., Nazek Alessa, Ngawang Namgyeland T. S. Karthik, 2021. MHD Flow of Thermally Radiative Maxwell Fluid Past a Heated Stretching Sheet with Cattaneo-Christov Dual Diffusion, Hindawi Journal of Mathematics, 2021. Article ID 5562667, 10 pages, https://doi.org/10.1155/2021/5562667 